\title{
Validación de una escala de clima organizacional en el sector de servicios en el noroeste de México
}

Jonathan Franco Betanzos

Martha Frías Armenta

Universidad de Sonora

\section{Resumen}

El objetivo del presente estudio es el de analizar la estructura factorial de una escala de Clima Organizacional para instituciones del sector servicio. Se encuestaron 116 sujetos, 39 pertenecían a un Centro de Salud dependiente de la Secretaria de Salud Pública del estado, 42 a un hospital privado y 35 a un hotel, todos de la ciudad de Hermosillo, Sonora, México. Los datos fueron analizados mediante un análisis factorial confirmatorio (EQS). El modelo hipotético establecía tres factores: estrés en el trabajo, comportamiento organizacional y organización en el trabajo. Sin embargo, los resultados mostraron que el instrumento evaluado conformó un solo factor basado en las dimensiones de adaptación, privación, desarrollo profesional, conocimiento de funciones, logro de objetivos, equidad, actitud de la organización, liderazgo, comunicación y trabajo en equipo. Se concluye que la estructura factorial propuesta no corresponde con la demostrada; sin embargo, arroja un instrumento validado que permite evaluar el Clima Organizacional en instituciones del Sector Servicio.

\section{Abstract}

The aim of this study was to analyze the factorial structure of the Organizational Climate Scale for the service sector institutions. The participants were 116 individuals; 39 were affiliated with a health center of the Public Health Secretary, 42 with a private hospital and 35 with a hotel, all of them at Hermosillo, Sonora, México. The data were analyzed by a confirmatory factor analysis (EQS).

The hypothetical model indicates three factors: organizational behavior, work stress and work organization. However, the results showed that the evaluated instrument confirmed only one factor based on the dimensions of adaptation, deprivation, professional development, knowledge of functions, achievement of goals, fairness, attitude of the organization, leadership, communication and team work. thus, it is concluded that the hypothesized factorial structure does not correspond with the data. Nevertheless, the validity and reliability of the instrument was proved and it allows the evaluation of the Organizational Climate in institutions of the Services sector.

Palabras clave: Estructura factorial, clima organizacional, validación, servicios, salud

Keywords: Factorial structure, organizational climate, validation, services, health 


\section{Introducción}

Las organizaciones contemporáneas luchan por su supervivencia y utilizan como estrategia el desarrollo constante para mantenerse en un nivel competitivo (Chiavenato, 2002). Dicha estrategia ha permitido la elaboración de diferentes métodos para la optimización de los recursos con el fin de ofrecer mejores productos o servicios (May Hofman, 2008). Las condiciones de estas se ven afectadas por las disposiciones contextuales en las que se desarrollan, que incluyen las relaciones entre los proveedores, los usuarios, los competidores, los trabajadores, los sindicatos, entre otras; así como el surgimiento de innovaciones, nuevos conocimientos, desarrollo tecnológico, regulaciones, políticas públicas y en general con cambios en el marco institucional (Melin, 1986).

Las organizaciones dedicadas a proporcionar servicios buscan, además, cubrir una necesidad específica para la cual han sido creadas. Dicha necesidad origina una complejidad adicional, por la cual la institución o empresa deberá trabajar para establecer su mejor rendimiento y satisfacer las necesidades de sus clientes o usuarios. Maslach (1993) afirma que las organizaciones dedicadas al sector servicio muestran una mayor dificultad para su adaptación a las diferentes exigencias del entorno; como consecuencia, sus trabajadores presentan padecimientos de tensión y estrés en el trabajo.

Dichas organizaciones son aquellas que no ofrecen un producto tangible (Bates et al., 2007), sino que ofrecen un servicio con el fin de satisfacer una necesidad específica del usuario. Entre ellas se encuentran: hospitales, hoteles, escuelas, etcétera (Instituto Mexicano de Normalización y Certificación A.C., 2001; Rodríguez, 1999).

Además de las características de la organización, Cifre (2008) afirma que la mayoría de los problemas de los trabajadores en su lugar de trabajo se derivan de factores ambientales, individuales y socio-demográficos, lo cual plantea que las características del trabajo son de suma importancia en el desempeño laboral y en el desarrollo individual de los trabadores. En la actualidad existen empresas o departamentos especializados en la optimización de las organizaciones, que buscan estrategias de intervención, y estas funcionan en algunos casos a partir de la percepción de los trabajadores (Chiavenato, 2006). Para este logro se han realizado mediciones del clima organizacional, que buscan identificar la percepción de los trabajadores en relación a diferentes aspectos del área de trabajo. Sin embargo, estas mediciones se han tomado de diferentes instrumentos, en donde la validez se basa en el concepto del instrumento mismo o de la variable a evaluar (Gan, 2005). Lo anterior implica que las evaluaciones de la percepción de los trabajadores se realicen a partir de instrumentos que carecen de validez y confiabilidad, lo cual pudiera ocasionar que las decisiones que se tomen a partir del mismo no correspondan con la realidad (Cabrera, 2006).

Un estudio realizado por parte de la Secretaria de Agricultura, Ganadería, Desarrollo Rural, Pesca y Alimentación del Gobierno Federal de México en el 2005 demuestra lo anteriormente expresado. Este estudio se basa en el diseño de un instrumento construido con reactivos de opción múltiple en forma dicotómica (Farfan et al, 2007). A partir de lo anterior, Rodríguez (1999) contrasta los diferentes estudios que se han realizado para tomar acciones en la búsqueda de mejorar el desempeño de una organización. Por una parte, se encuentran los estudios destinados a la gestión de la calidad de los productos o servicios que se ofertan, estableciendo el compromiso de determinar y gestionar que el ambiente de trabajo tenga una influencia positiva en la motivación, la satisfacción y el ejercicio del personal con el propósito de mejorar el desempeño de la organización(Montaño, 2003). Por otra parte, se encuentran los estudios destinados al modelo de trabajo del desarrollo organizacional que buscan satisfacer necesidades intrínsecas 
de sus miembros; ya sean económicas, de desarrollo personal, emocionales, intelectuales, entre otras (Chiavenato, 2000; Myers, 2000).

Según el Instituto Mexicano de Normalización y Certificación A.C.(2001), en la norma 9001:2000 es requisito de las organizaciones el determinar, recopilar y analizar los datos apropiados para demostrar la idoneidad y eficacia de la misma, con el fin de realizar mejoras. A partir de lo anterior, Anderson, Sweeny y Williams (2005) afirman que las organizaciones que tienen éxito son las que comprenden la información y la usan eficazmente.

No obstante, en la consultoría moderna existe una gran demanda de estudios entre los cuales el clima organizacional representa una prioridad, lo cual, según Moreno (2001), tiene una explicación sistémica concerniente a los factores externos en los cuales la organización se encuentra inmersa, así como los procesos definidos y los resultado obtenidos, siendo este tipo de estudios el que las organizaciones determinan como necesarios para obtener un mejor rendimiento. Sin embargo, los instrumentos con los cuales se realizan estos estudios no siempre muestran validez y confiabilidad. Algunos de ellos establecen una escala dicotómica, otras no reportan las características psicométricas de los instrumentos (Del Castillo, Acosta \& Montes 2008).

A pesar de la importancia otorgada a los métodos cuantitativos en la investigación social y del comportamiento, en la mayoría de las ocasiones no se aprovechan cabalmente los recursos que ofrecen (Corral et al., 2001). Maish (2007) afirma que el aspecto instrumental de la investigación relacionada con la metodología de estudios de Clima Organizacional normalmente responde a la elección de las técnicas e instrumentos que resulten más pertinentes para recoger la información necesaria. Esto puede perjudicar a la toma de decisiones, ya que no necesariamente se cumple con la validez y la confiabilidad necesarias. Según Meliá (2008) los instrumentos de medición de variables psicosociales deben estar fundamentados en una metodología integral con soporte científico, tanto en las características de la población a aplicar como en la psicometría adecuada y estandarizada, además de cumplir con los requisitos de confiabilidad y validez, que permitan ofrecer a las organizaciones y a los profesionales indicadores certeros de evaluación para la toma de decisiones.

Según Gans (1999) la falta de una apropiada metodología en la interpretación de datos para la toma de decisiones puede afectar sustancialmente en la implementación de la estrategia a seguir. A su vez Hudd, Dumalao y Erdman-Sager (2000) afirman que la existencia de valores cuantitativos no necesariamente significa que la variable fue correctamente definida, para lo cual es necesario tomar las medidas estadísticamente necesarias.

Valenzuela, Montenegro, Valenzuela y Alvarado (2007) afirman que cualquier medición que se emplee en la evaluación psicológica debe ser confiable, de lo contrario resulta inútil, ya que los instrumentos deben medir aquello para lo que están diseñados. A partir de lo anterior, se reconoce la importancia de diseñar instrumentos de medición enfocados a los sectores productivos correspondientes, de tal forma que permitan la toma de decisiones basadas en las variables que la organización identifica como apropiadas y relacionadas con la labor diaria. Por tal motivo, se pretende ofrecer un instrumento que evalué las variables asociadas al desempeño del trabajador y la percepción que tiene sobre el área de trabajo, para lo cual se tomarán en cuenta las variables relacionadas con los factores concernientes al comportamiento de la organización, la organización del trabajo y el estrés laboral.

La validación de una Escala de Clima Organizacional en el sector servicios permite ofrecer una unidad de medida que pueda determinar cuáles son las variables a considerar en una intervención, reestructura o toma de algunas disposiciones con respecto a la percepción de los trabajadores que laboren en 
ese sector. El clima organizacional surge de la necesidad de reconocer los fenómenos globales que tienen lugar dentro de las instituciones laborales desde una perspectiva general, pero al mismo tiempo, este constructo requiere que sea lo suficientemente simple como para poder servir de orientación a trabajos prácticos de intervención en las corporaciones, como lo establece el modelo del Desarrollo Organizacional (Guillen et al., 2000; Guizar 2001).

$\begin{array}{cccc}\text { Rodríguez } & (1999) & \text { define el Clima } \\ \text { Organizacional } & \text { como } & \text { un } & \text { concepto }\end{array}$
multidimensional que implica una referencia constante de los miembros respecto a la organización, enfocándose en aquellas variables ambientales internas. Para el estudio del Clima Organizacional es necesaria la comprensión de las variables ambientales internas que afectan el comportamiento de los individuos en la organización. Esto se puede llevar a cabo a través de un estudio de las percepciones que los individuos tienen de ellas. Las variables se pueden clasificar en: "Ambiente físico", tales como el espacio, condiciones de ruido, calor, contaminación, instalaciones, maquinaria, entre otras (Pinazo et al., 2001; Rodriguez,1999); "Estructurales" (Chiavenato, 2000) como el tamaño de la organización, estructura formal, estilo de dirección, etcétera ; "Ambiente social", que incluyen al compañerismo, conflictos entre personas o departamentos, comunicaciones, etcétera; "Personales", como son las aptitudes, actitudes, motivaciones, expectativas, y otras más (Peiró et al., 2002); "Propias del comportamiento organizacional," que comprenden la productividad, ausentismo, rotación, satisfacción laboral, tensiones y estrés, entre otras (Schmidt et al., 2001). Todas estas variables configuran el clima de una organización a través de la percepción que tienen los miembros de ellas.

Por tal motivo, el objetivo del presente estudio fue medir la estructura factorial del Instrumento de Clima Organizacional, el cual está construido para utilizarlo en instituciones del sector servicio a partir de lo definido por Peiró et al., (2002); Chiavenato, (2000); Schmidt et al., (2001) Robbins (2004); Pinazo et al., (2001).

\section{Método}

\section{Participantes}

Se encuestaron a 116 sujetos en la ciudad de Hermosillo, Sonora, México: 39 pertenecían a un Centro de Salud dependiente de la Secretaria de Salud Pública del Estado, 42 a un hospital privado y 35 personas que laboraban en un hotel. De todos ellos 33 fueron hombres y 83 mujeres. La Tabla 1 muestra las características de la población del estudio.

\begin{tabular}{|c|c|c|}
\hline Variable & $\mathrm{N}$ & $\%$ \\
\hline Masculino & 33 & $28.45 \%$ \\
\hline Femenino & 83 & $71.55 \%$ \\
\hline \multicolumn{3}{|l|}{ Nivel de educación } \\
\hline Menor de Preparatoria & 38 & $32.76 \%$ \\
\hline Preparatoria & 22 & $18.97 \%$ \\
\hline Nivel Técnico & 31 & $26.72 \%$ \\
\hline Profesionista & 25 & $21.55 \%$ \\
\hline \multicolumn{3}{|c|}{ Área de trabajo Centro de Salud SSP } \\
\hline Dirección & 2 & $5.13 \%$ \\
\hline Medicina General & 4 & $10.26 \%$ \\
\hline Odontología & 2 & $5.13 \%$ \\
\hline Epidemiología & 2 & $5.13 \%$ \\
\hline Estadística & 2 & $5.13 \%$ \\
\hline Enfermería & 12 & $30.77 \%$ \\
\hline Trabajo Social & 2 & $5.13 \%$ \\
\hline Promoción de Salud & 3 & $7.69 \%$ \\
\hline Administración & 9 & $23.08 \%$ \\
\hline Servicios Generales & 1 & $2.56 \%$ \\
\hline \multicolumn{3}{|c|}{ Área de trabajo hospital privado } \\
\hline Administración & 12 & $28.57 \%$ \\
\hline Cocina y Nutrición & 6 & $14.29 \%$ \\
\hline Apoyo a Servicios & 16 & $38.10 \%$ \\
\hline Recepción & 4 & $100.00 \%$ \\
\hline Enfermería & 4 & $9.52 \%$ \\
\hline \multicolumn{3}{|l|}{ Área de trabajo de hotel } \\
\hline Camaristas y Limpieza & 8 & $22.86 \%$ \\
\hline Mantenimiento & 5 & $14.29 \%$ \\
\hline Administración & 5 & $14.29 \%$ \\
\hline Restaurante y Cocina & 11 & $31.43 \%$ \\
\hline Recepción & 6 & $17.14 \%$ \\
\hline
\end{tabular}




\section{Instrumento}

Seconformó la escala de clima organizacional con 116 afirmaciones, las cuales se distribuyen en tres factores:

1) El factor de estrés laboral, compuesto por las dimensiones de adaptación, privación y frustración, las cuales buscan esclarecer la sensación de los trabajadores en ámbitos relacionados con el conocimiento de los usuarios a los que se atiende, el trabajo bajo presión que realizan, el desafío que éste les impone y la estabilidad laboral que ellos perciben (Peiró et al., 2002).

2) El factor de organización del trabajo, que se definió por las dimensiones de equidad, remuneración, logro de objetivos, conocimiento de funciones, desarrollo profesional e instalaciones y herramientas, las cuales buscan conocer la sensación de los trabajadores en ámbitos relacionados a la definición de la tarea, la retribución, distribución de la carga de trabajo, infraestructura física y la participación que tienen para el desarrollo de la organización (Chiavenato, 2000; Schmidt et al., 2001).

3) El factor de comportamiento de la organización, se conforma por las dimensiones de actitud hacia la organización, liderazgo, comunicación, trabajo en equipo e identidad. Estos buscan analizar la percepción de los trabajadores en la eficacia de la comunicación en la organización, la forma en que se solucionan los problemas, como son abordados y la identificación que estos tienen hacia la organización (Robbins, 2004; Pinazo et al., 2001).

\section{Procedimiento.}

En cada una de las organizaciones se detuvieron las actividades de las diferentes áreas de trabajo para la aplicación del instrumento, por lo tanto, este fue administrado sin interrupciones en un área acondicionada dentro de la misma organización, en la que se separaron los participantes en grupos de 10 personas con el fin de mejorar el manejo del grupo. Las encuestas fueron aplicadas por estudiantes de licenciatura en psicología, teniendo como duración en promedio 20 minutos.

\section{Análisis de datos.}

En primera instancia se obtuvieron las estadísticas univariadas, es decir, medias para variables continuas y frecuencias para las variables categóricas. A su vez se calcularon alfas de Cronbach para todas las escalas con el fin de determinar su confiabilidad (consistencia interna). Se crearon índices, los cuales se calcularon promediando las respuestas de las variables dentro de cada dimensión. Además, se llevó a cabo un análisis factorial confirmatorio con el paquete estadístico EQS utilizando un modelo de relaciones estructurales (Bentler, 1995) congregando en factores las dimensiones según lo estipulado, con base en lo definido por varios autores (Pinazo et al., 2001; Chiavenato, 2000; Peiróet al., 2002; Schmidtet al., 2001).

Para determinar la pertinencia del modelo se utilizaron indicadores de bondad de ajuste. Se esperaba que el indicado estadístico $X^{2}$, que determina la diferencia entre el modelo hipotético y el real, no fuera significativo. Además, se utilizaron el índice Bentler - Bonett de Ajuste No Normado y el Índice de Ajuste Comparativo. El nivel mínimo aceptable de dichos indicadores es de .90 (Bentler, 1993).

Se conformó un modelo consistente en los 3 factores antes mencionados: estrés laboral, comportamiento de la organización y organización en el trabajo. 


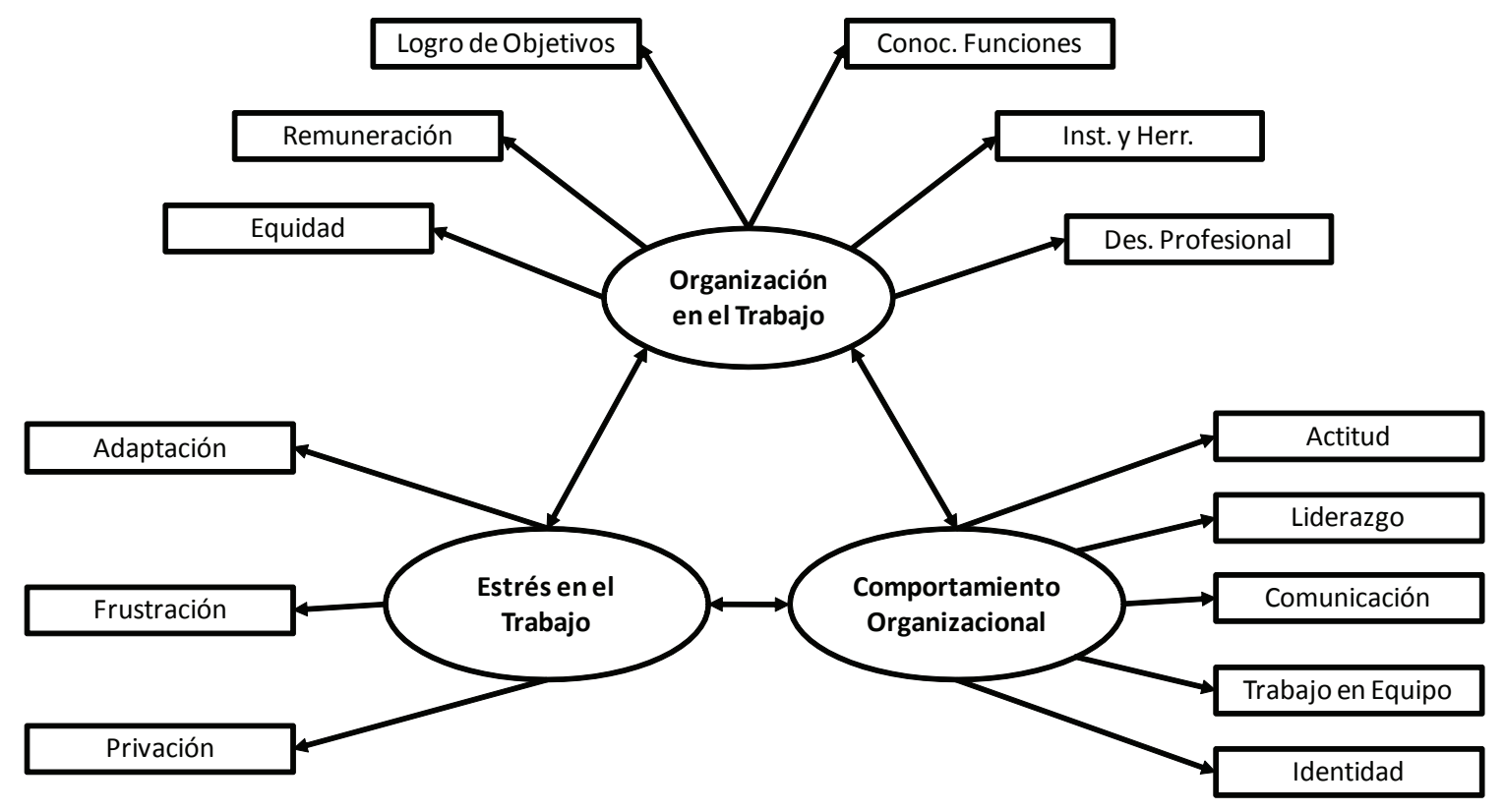

Figura 1. Modelo Hipotético Tres Dimensiones de la Escala de Clima Organizacional

A partir de los factores expresados por Eysenck y Eysenck (1987) se pretendió integrar los factores propuestos en un constructo de segundo orden que surge de las interrelaciones entre los primeros, y que configura las dimensiones básicas, caracterizadas por su estabilidad y consistencia (Escolano et al., 2000). Este modelo se especificó tal y como se muestra en la Figura 2.

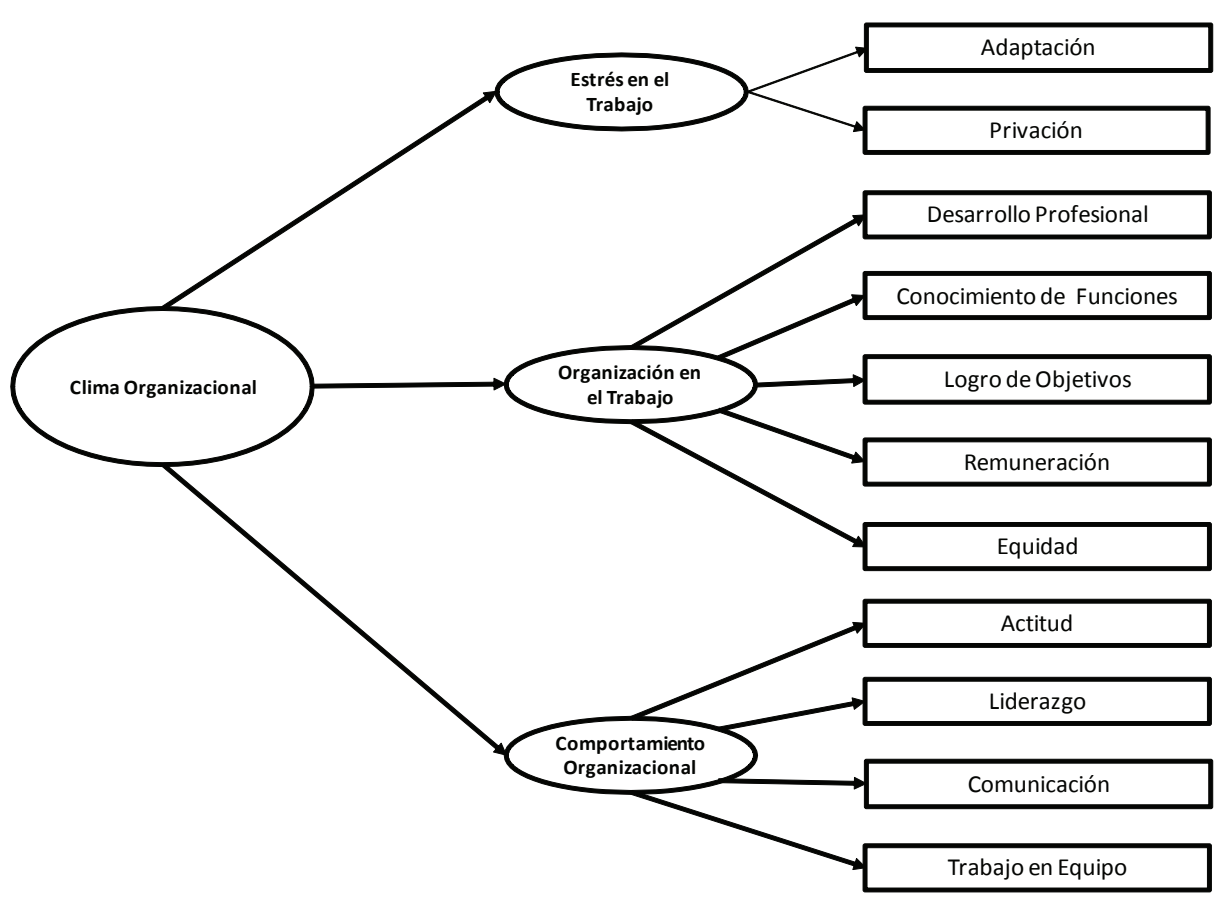

Figura 2. Modelo Hipotético de la Escala de Clima Organizacional con Factor de Segundo Orden. 


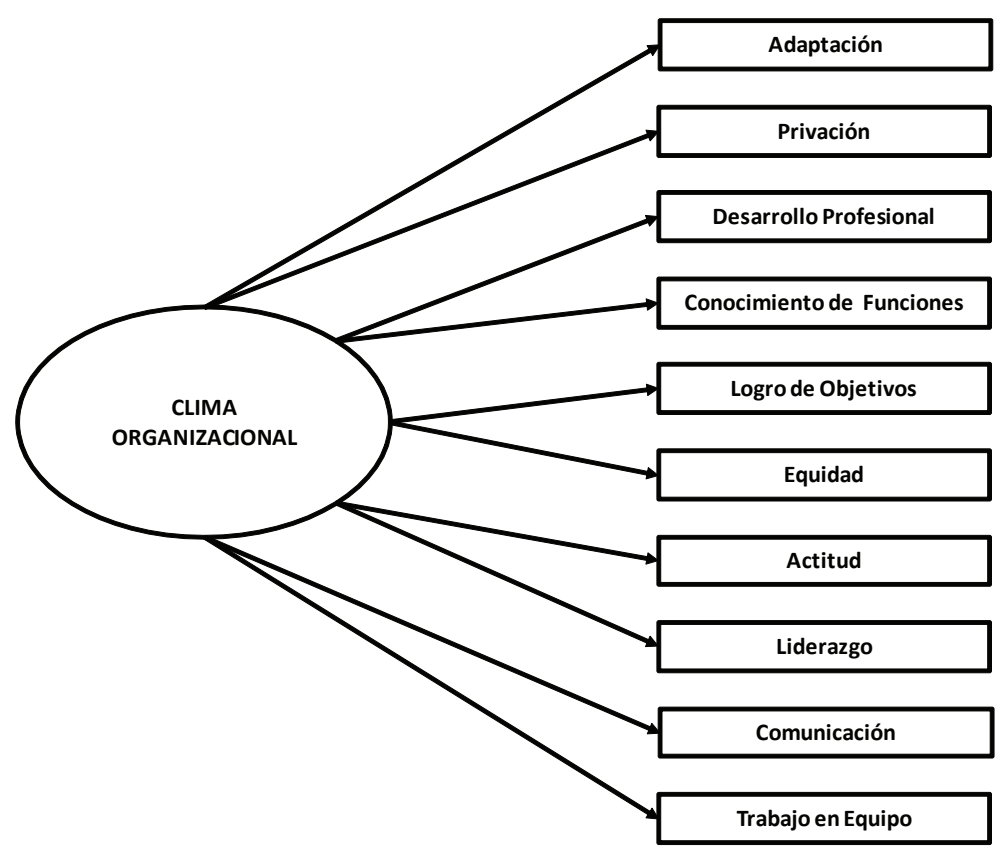

Figura 3. Modelo Hipotético de la Escala de Clima Organizacional.

Finalmente se llevó a cabo un análisis factorial relacionando las dimensiones directamente, con un solo factor de Clima Organizacional, esto con el propósito de probar el modelo más parsimonioso, que permita a los usuarios del instrumento un fácil manejo y visualización (Fierro, 2000), el resultado se muestra en la Figura 3.

\section{Resultados}

Resultante de lo anterior, la escala de Clima Organizacional aplicada en organizaciones del sector servicio en la Ciudad de Hermosillo Sonora, queda con un coeficiente de confiabilidad de alfa $=.90$, distribuyéndose en 10 dimensiones compuestas por 59 reactivos, distribuidos como se muestra en la Tabla 2.

Tabla 2. Descripción de la Escala de Clima Organizacional

\begin{tabular}{|c|c|c|c|c|c|c|}
\hline Variable & $\mathbf{N}$ & Media & D.E. & Min & Max & Alfa \\
\hline Adaptación & & & & & & 0.607 \\
\hline $\begin{array}{l}\text { Es común recibir comentarios positivos de nuestros usuarios con } \\
\text { respecto a los servicios brindados. }\end{array}$ & 114 & 3.78 & 1.011 & 1 & 5 & \\
\hline Mi actitud ante los usuarios generalmente es cálida y amistosa. & 116 & 4.56 & 0.65 & 2 & 5 & \\
\hline La imagen de quienes dan servicio, reflejan confianza y calidad. & 114 & 4.18 & 0.79 & 2 & 5 & \\
\hline Conozco con precisión las necesidades de los usuarios. & 113 & 4.07 & 0.853 & 1 & 5 & \\
\hline $\begin{array}{l}\text { La prioridad de la Institución es brindar un buen servicio a los } \\
\text { usuarios. }\end{array}$ & 114 & 4.75 & 0.525 & 2 & 5 & \\
\hline $\begin{array}{l}\text { Las quejas y sugerencias de los usuarios siempre son tomadas en } \\
\text { cuenta para mejorar el servicio. }\end{array}$ & 110 & 4.17 & 1.148 & 1 & 5 & \\
\hline
\end{tabular}


Tabla 2. Descripción de la Escala de Clima Organizacional

\begin{tabular}{|c|c|c|c|c|c|c|}
\hline Variable & $\mathbf{N}$ & Media & D.E. & Min & Max & Alfa \\
\hline Privación & & & & & & 0.679 \\
\hline $\begin{array}{l}\text { En la ejecución de mi trabajo diario, la calidad tiene la más alta } \\
\text { prioridad. }\end{array}$ & 111 & 4.57 & 0.696 & 2 & 5 & \\
\hline Los objetivos de mi puesto son razonablemente alcanzables. & 113 & 4.19 & 0.714 & 2 & 5 & \\
\hline $\begin{array}{l}\text { La planeación y coordinación de nuestro trabajo nos ubica como un } \\
\text { área competitiva. }\end{array}$ & 112 & 4.04 & 0.977 & 1 & 5 & \\
\hline $\begin{array}{l}\text { En mi trabajo me encuentro con desafíos constantes, lo cual me } \\
\text { motiva a seguir trabajando. }\end{array}$ & 113 & 3.45 & 1.165 & 1 & 5 & \\
\hline Siempre me imponen nuevas metas para mi trabajo. & 111 & 4.34 & 0.792 & 1 & 5 & \\
\hline Los desafíos que se me imponen en el trabajo, me ayudan a mejorar & 112 & 3.96 & 0.92 & 1 & 5 & \\
\hline
\end{tabular}

\section{Desarrollo Profesional}

Mi trabajo actual me ayuda a lograr mis objetivos personales.

El potencial profesional se desarrolla constantemente en nuestro trabajo.

Siempre muestras interés por desarrollarnos personal y profesionalmente.

Se dedica el tiempo necesario al entrenamiento y desarrollo personal de cada uno de nosotros.

Pienso que me puedo desarrollar y crecer profesionalmente en esta institución

Al realizar mis actividades laborales voy creciendo personal y profesionalmente.

\section{Conocimiento de Funciones}

Mi equipo de trabajo conoce perfectamente lo que tiene que hacer.

Los resultados esperados en mi trabajo están muy claramente definidos.

En nuestra área de trabajo cada quien tiene definida su función, no se repite con las de los demás.

Cuento con suficiente información formal para tomar mis decisiones.

Todos mis compañeros tienen conocimiento sobre el trabajo que nos compete.

\section{Logro de Objetivos}

En mi área de trabajo estamos orientados a la obtención de resultados.

Los objetivos de trabajo están definidos claramente.

Las metas de trabajo son objetivas y realistas.

Existen metas definidas para las actividades que realizamos.
0.628

$\begin{array}{lllll}114 & 3.97 & 0.954 & 1 & 5 \\ 111 & 3.97 & 0.909 & 1 & 5 \\ 113 & 4.45 & 0.876 & 1 & 9 \\ 111 & 3.52 & 1.212 & 1 & 5 \\ 114 & 3.7 & 1.323 & 1 & 5 \\ 112 & 4.21 & 1.026 & 1 & 5\end{array}$

0.641

$\begin{array}{lllll}113 & 4.56 & 0.611 & 3 & 5 \\ 112 & 4.27 & 0.782 & 1 & 5 \\ 113 & 3.65 & 1.116 & 1 & 5 \\ 114 & 3.98 & 1.039 & 1 & 5 \\ 113 & 4.21 & 0.94 & 1 & 5\end{array}$

\subsection{5}

$\begin{array}{lllll}110 & 4.06 & 1.136 & 1 & 5 \\ 114 & 4.2 & 0.914 & 1 & 5 \\ 114 & 4.06 & 0.875 & 1 & 5 \\ 112 & 3.91 & 1.119 & 1 & 5\end{array}$


Tabla 2. Descripción de la Escala de Clima Organizacional

\begin{tabular}{|c|c|c|c|c|c|c|}
\hline Variable & $\mathbf{N}$ & Media & D.E. & Min & Max & Alfa \\
\hline $\begin{array}{l}\text { En mi área de trabajo existen objetivos determinados para nuestro } \\
\text { trabajo. }\end{array}$ & 110 & 4.05 & 0.892 & 1 & 5 & \\
\hline Siempre trabajamos buscando cumplir con los objetivos que existen. & 113 & 4.37 & 0.793 & 1 & 5 & \\
\hline Equidad & & & & & & 0.601 \\
\hline En mi área de trabajo no existen preferencias por nadie. & 114 & 3.07 & 1.308 & 1 & 5 & \\
\hline Mis compañeros y yo tenemos la misma carga de trabajo. & 114 & 3.46 & 1.13 & 1 & 5 & \\
\hline $\begin{array}{l}\text { Los directivos nos ven a todos como iguales, sin preferencias por } \\
\text { nadie. }\end{array}$ & 113 & 3.68 & 1.071 & 1 & 5 & \\
\hline La asignación de las tareas extras siempre es justa. & 111 & 3.6 & 1.162 & 1 & 5 & \\
\hline En la institución no existen preferencias por nadie. & 113 & 3.06 & 1.182 & 1 & 5 & \\
\hline Mi departamento se caracteriza por la equidad que existe en él. & 112 & 3.9 & 0.93 & 1 & 5 & \\
\hline Actitud de la Organización & & & & & & 0.795 \\
\hline Siento que la dirección tiene buena actitud hacia los trabajadores. & 112 & 4.07 & 0.965 & 1 & 5 & \\
\hline $\begin{array}{l}\text { La actitud que muestran los directivos de la institución se proyecta } \\
\text { hacia todos los empleados. }\end{array}$ & 113 & 3.73 & 1.088 & 1 & 5 & \\
\hline $\begin{array}{l}\text { La dirección afronta adecuadamente las actitudes negativas por parte } \\
\text { de los empleados. }\end{array}$ & 113 & 3.74 & 1.084 & 1 & 5 & \\
\hline La institución muestra una actitud favorable para los usuarios. & 112 & 4.21 & 0.915 & 1 & 5 & \\
\hline $\begin{array}{l}\text { Siento que la institución esta comprometida con sus empleados y con } \\
\text { su trabajo. }\end{array}$ & 111 & 3.89 & 0.888 & 1 & 5 & \\
\hline $\begin{array}{l}\text { La dirección afronta adecuadamente las problemáticas que se } \\
\text { enfrenta. }\end{array}$ & 113 & 4.16 & 0.922 & 1 & 5 & \\
\hline Liderazgo & & & & & & 0.776 \\
\hline $\begin{array}{l}\text { Siempre que me piden objetivos mi jefe le da seguimiento a su } \\
\text { realización. }\end{array}$ & 109 & 3.93 & 1.043 & 1 & 5 & \\
\hline Los superiores buscan la opinión del personal. & 113 & 3.38 & 1.175 & 1 & 5 & \\
\hline Hay congruencia entre lo que piden y hacen mis jefes. & 110 & 3.78 & 1.053 & 1 & 5 & \\
\hline Mi jefe del equipo se adapta rápidamente su estilo a los cambios. & 113 & 3.82 & 0.975 & 1 & 5 & \\
\hline Siempre que logro un buen resultado, mi jefe reconoce mi aportación. & 110 & 3.06 & 1.329 & 1 & 5 & \\
\hline $\begin{array}{l}\text { Mi jefe siempre pregunta sobre cosas nuevas para mejorar nuestro } \\
\text { trabajo. }\end{array}$ & 113 & 3.45 & 1.165 & 1 & 5 & \\
\hline Comunicación & & & & & & 0.694 \\
\hline $\begin{array}{l}\text { Estoy oportunamente informado de lo que sucede en mi área de } \\
\text { trabajo. }\end{array}$ & 111 & 4.15 & 0.865 & 1 & 5 & \\
\hline $\begin{array}{l}\text { La comunicación con mi jefe(a) me ayuda a entender la dinámica de } \\
\text { mi área de trabajo. }\end{array}$ & 113 & 4.13 & 1.048 & 1 & 5 & \\
\hline La comunicación formal es adecuada y suficiente. & 113 & 3.73 & 0.866 & 1 & 5 & \\
\hline
\end{tabular}


Tabla 2. Descripción de la Escala de Clima Organizacional

\begin{tabular}{|c|c|c|c|c|c|c|}
\hline Variable & $\mathbf{N}$ & Media & D.E. & Min & Max & Alfa \\
\hline $\begin{array}{l}\text { Me mantengo informado sobre el trabajo que se realiza en mi área de } \\
\text { trabajo. }\end{array}$ & 115 & 4.3 & 0.837 & 1 & 5 & \\
\hline $\begin{array}{l}\text { Yo puedo decir libremente a mi jefe cuando estoy en desacuerdo con } \\
\text { él. }\end{array}$ & 113 & 3.96 & 1.191 & 1 & 5 & \\
\hline La comunicación es adecuada en la organización. & 112 & 3.87 & 0.925 & 1 & 5 & \\
\hline Trabajo en Equipo & & & & & & 0.725 \\
\hline $\begin{array}{l}\text { Mis compañeros y yo convivimos amenamente dentro o fuera del } \\
\text { trabajo. }\end{array}$ & 112 & 3.98 & 1.022 & 1 & 5 & \\
\hline Mis compañeros se comprometen con el trabajo. & 114 & 3.8 & 0.979 & 1 & 5 & \\
\hline Entre compañeros damos retroalimentación de nuestro trabajo. & 114 & 3.65 & 1.03 & 1 & 5 & \\
\hline $\begin{array}{l}\text { Entre compañeros siempre buscamos información para ayudar a los } \\
\text { otros en algunas problemáticas. }\end{array}$ & 114 & 3.64 & 1.114 & 1 & 5 & \\
\hline Mis compañeros se escuchan activamente mientras otros hablan. & 112 & 3.53 & 1.048 & 1 & 5 & \\
\hline Tenemos buena comunicación en el equipo. & 113 & 4.08 & 0.992 & 1 & 5 & \\
\hline
\end{tabular}

El análisis factorial confirmatorio de la Escala de Clima Organización que probó el modelo hipotético de tres factores, basado en lo propuesto por Peiró et al., (2002); Chiavenato
(2000); Schmidt et al.,(2004) y Pinazo y Carrero (2001), muestra una alta interrelación muy pronunciada entre los tres factores especificados (ver Figura 4).

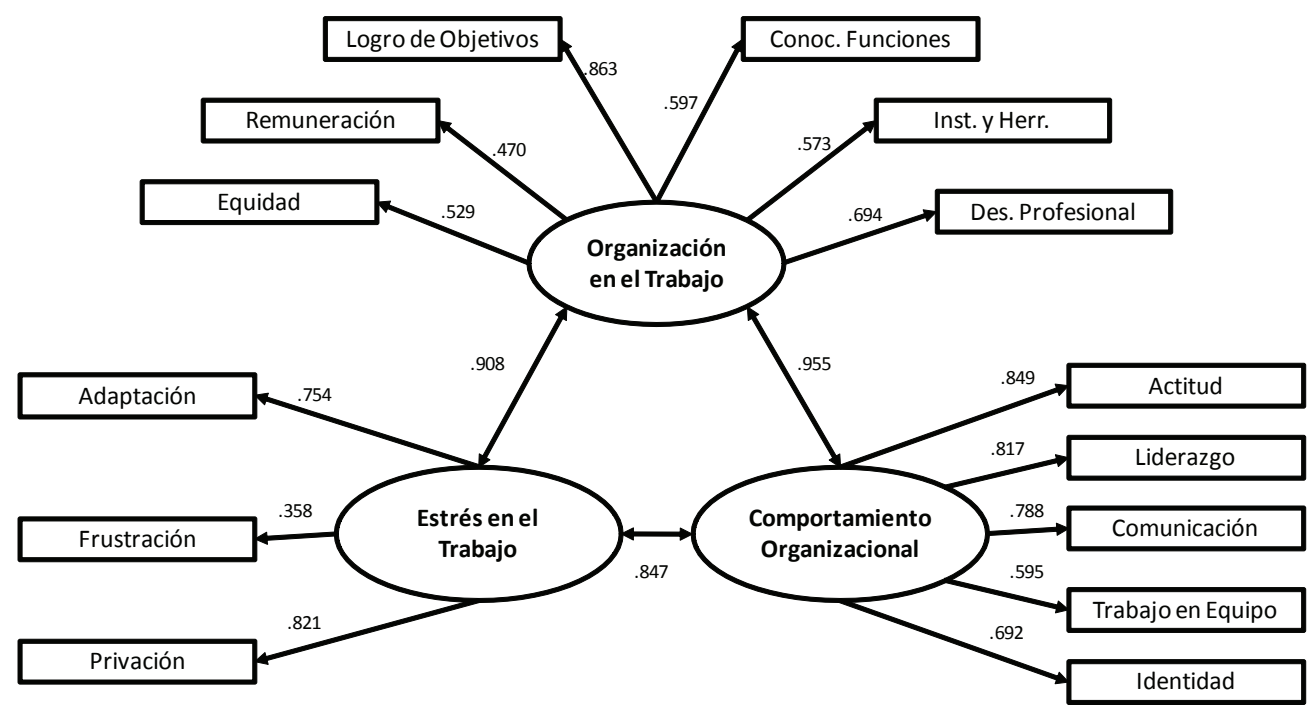

$X^{2}=72$, G.L. $=41, p=0.001$, B.B.N. $=0.893$, B.B.N.F.I. $=0.932$, C.F.I. $=0.950$, R.M.S.E.A. $=0.08$

Figura 4. Modelo Escala de Clima Organizacional con tres factores. 
Para buscar coherencia entre los resultados dada la poca discriminación entre factores del modelo anterior, se llevó a cabo otro análisis probandolos tresfactores, peroomitiendoaquellas dimensiones que no guardaban consistencia a partir de los coeficientes de confiabilidad de los factores, agrupándolos en: estrés en el trabajo, organización en el trabajo y comportamiento de la organización en un factor de segundo orden denominado clima organizacional (Eysenck y Eysenck, 1987).
Los resultados del modelo de la Escala de Clima Organizacional con Factor de Segundo Orden con sus respectivos pesos factoriales se muestran en la Figura 5, resultando significativos los índices de correlación existentes entre las dimensiones anteriormente expresadas y el factor de segundo orden en la que se muestra un valor mínimo de correlación de 0.904 con estrés en el trabajo y una correlación de 1.00 con organización en el Trabajo.

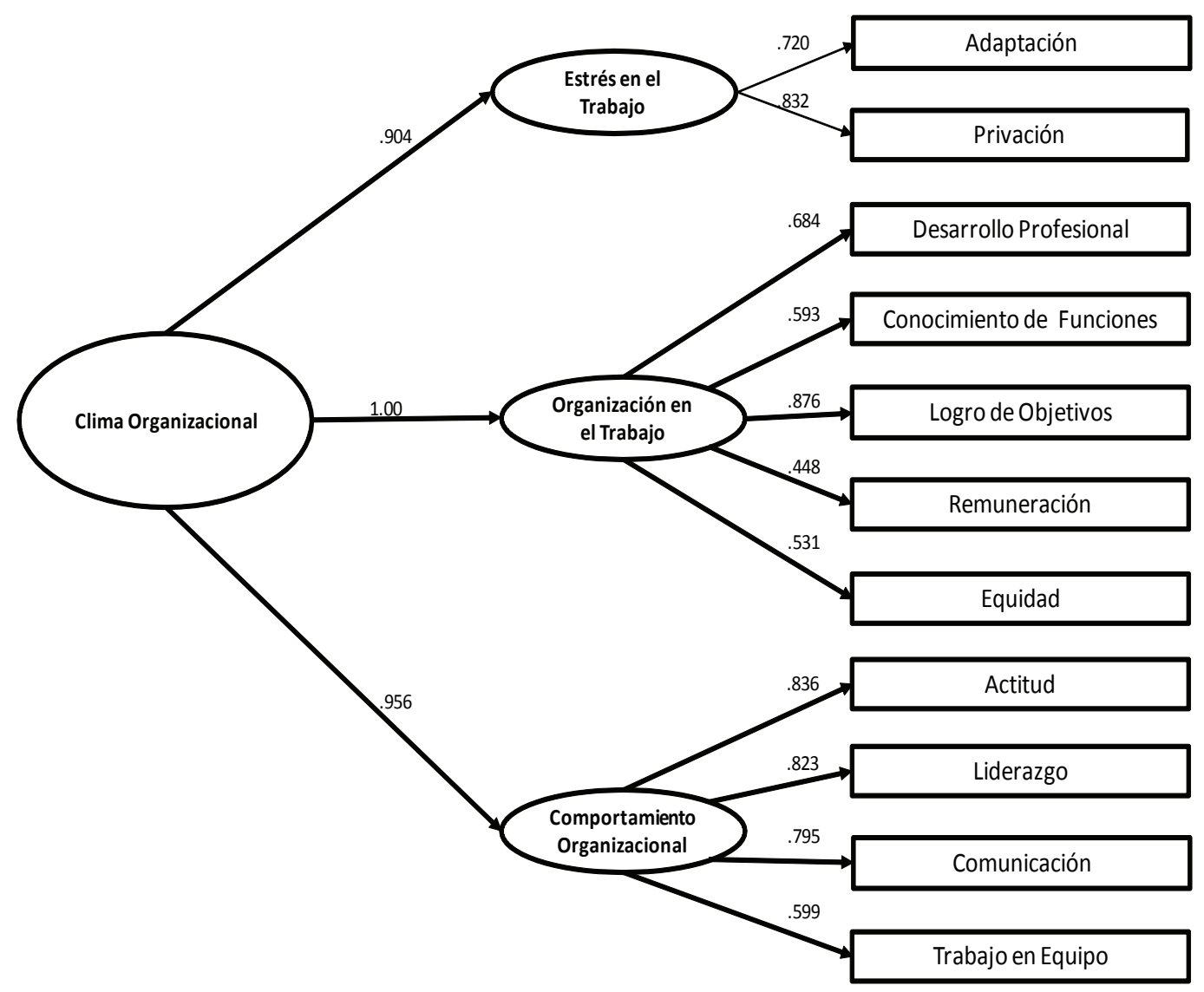

$X^{2}=73, G . L .=40, p=0.0009$, B.B.N. $=0.891$, B.B.N.F.I $=0.925$, C.F.I $=0.947$, R.M.S.E.A. $=0.08$

Figura 5. Modelo de la Escala de Clima Organizacional con Factor de Segundo Orden. 


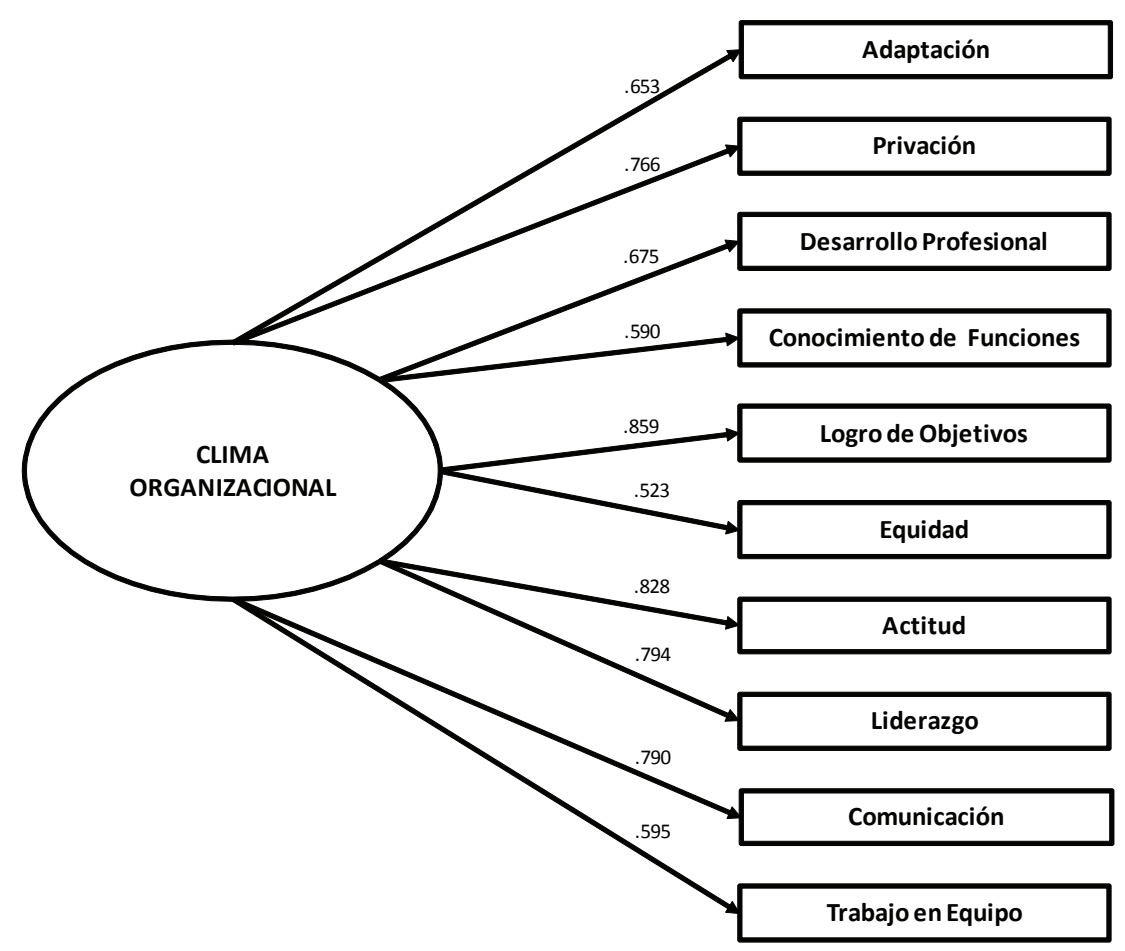

$\mathrm{X}^{2}=81, \mathrm{G} . \mathrm{L} .=44, \mathrm{p}=0.0005$, B.B.N. $=0.880$, B.B.N.F.I $=0.925$, C.F.I $=0.940$,

R.M.S.E.A. $=0.08$

Figura 6. Modelo de la Escala de Clima Organizacional

Considerando que estos resultados replicaban la poca discriminación producida por el primer modelo, la representación de la Escala de Clima Organizacional como factor de segundo orden se omitió del modelo final, el cual se basó en un solo factor, mismo que se muestra en la Figura 6.

\section{Discusión}

Los resultados mostraron que el instrumento propuesto para medir el clima organizacional en instituciones del sector servicio no mostró la estructura factorial propuesta, es decir, integrada por los tres factores planteados. Esto parece reflejar una falta de correspondencia entre los expuesto por los encuestados y el modelo propuesto por los autores referidos en este trabajo.

Es importante mencionar que otros problemas surgieron en la especificación de los modelos. En el caso del factor de estrés en el trabajo, formado de acuerdo a lo definido por Peiró (1999) García et al.,(2002) - donde se ubicaban las dimensiones de adaptación, frustración y privación- no se formó coherentemente. El factor se conformó solamente al eliminar la variable de frustración, ya que la población a la que se aplicó no lo consideraba como un factor asociado al estrés laboral. Por otra parte, el factor de organización en el trabajo, definido a partir de lo mencionado por Chiavenato (2000), y Schmidt, Shulla y Schmitt (2001) - compuesto por las variables de equidad, remuneración, logro de objetivos, conocimiento de funciones, desarrollo profesional e instalaciones y herramientas tampoco mostró ser congruente con la teoría, ya que esta última variable fue eliminada en el análisis, debido a su poca en relación con el factor propuesto. Esto se puede deber a que en las diferentes organizaciones, se percibió de manera diferente esta variable, dadas las 
diferencias entre las instituciones con respecto a las instalaciones y herramientas donde y con las cuales fueron administrados los instrumentos. En el factor denominado comportamiento de la organización, definido a partir de lo mencionado por Robbins (2004) y Pinazo et al., (2001) construido por las dimensiones de actitud, liderazgo, comunicación, trabajo en equipo e identidad, se encontró que esta última no mostró relación con las demás variables. Esto probablemente se deba a las características de la variable, que buscaba identificar aspectos personales, mientras que las demás se relacionaban con la percepción de los trabajadores de los diferentes aspectos de la organización.

Los resultados mostraron covarianzas muy altas entre los factores, inclusive más altas que los pesos factoriales entre cada constructo y sus indicadores, lo que indica una falta de validez discriminante. Esto implica que los factores no se distingan entre sí, lo que nos sugirió que era posible formar un factor de segundo orden o un factor único subyacente a las interrelaciones entre todos los reactivos del instrumento.

Considerando lo anterior, como segunda parte del análisis, se intentó integrar los factores de estrés en el trabajo, organización del trabajo y comportamiento de la organización dentro de un factor de segundo orden según lo especificado por Eysenck y Eysenck (1987), donde para la comprensión de fenómenos del comportamiento complejo se incluyen factores de primer orden, semejantes a los llamados de segundo orden. Sin embargo, a partir de los resultados, los altos coeficientes estructurales indicaron la existencia de un factor único, donde los denominados factores de primer orden, para este modelo (estrés en el trabajo, organización del trabajo y comportamiento de la organización) no tienen razón de existir, ya que el modelo puede ser conformado por las dimensiones de adaptación, privación, desarrollo profesional, conocimiento de funciones, logro de objetivos, equidad, actitud, liderazgo, comunicación y trabajo en equipo, integrándose todas estas en el factor de clima organizacional, Esto indica el carácter unidimensional de la escala conformada.

A partir de lo anterior, se considera pertinente concluir que la escala de clima organizacional, como factor unitario presenta validez de constructo y confiabilidad estadísticamente comprobada, a diferencia de estudios con escalas previamente propuestas, los que solo muestran resultados descriptivos y no presentan la confiabilidad y validez de los instrumentos aplicados. No obstante, es importante analizar en futuros estudios porqué algunas variables que se establecían teóricamente como pertenecientes a los constructos planteados no se conformaron dentro de los factores probados en el modelo estructural.

La definición de esta escala permite identificar aquellos factores asociados a la integración del clima organizacional de instituciones del sector servicio, tanto en el sector público, como en el privado, permitiendo tener un marco de referencia para futuras investigaciones (Del Castillo y Cols., 2008) o bien, para la toma de decisiones dentro de las organizaciones (Molina, 2005). Además, proporcionaría una medición válida y confiable de los factores que intervienen en los procesos de adaptación de los trabajadores a las actividades propias de servicio. Esto ayudaría a las empresas a analizar las variables que pudieran mejorar el clima, lo que repercutiría en el mejoramiento de los servicios que proporcionan.

Derivado de la complejidad del concepto de clima organizacional (Robbins, 1999; Robbins, 2004), es importante señalar que para este estudio se consideraron aquellos factores apropiados a la población y al sector de aplicación. Sin embargo, es necesario que para la utilización de este instrumento en futuras aplicaciones, se indague la adaptación y estandarización del mismo en otras poblaciones con el fin de validar sus resultados (Molina, 2005), y de que estos puedan ser utilizados para la toma de decisiones. 
Definir el clima organizacional a partir de variables mesurables permite, además de evaluarla ser congruentes con la percepción que los trabajadores tienen de las instituciones o empresas del sector servicio de la región. La intención del presente estudio, además de ofrecer una herramienta de medición valida y confiable al ámbito laboral, fue la de ofrecer un panorama de la utilización de las herramientas estadísticas para la medición de las características psicométricas, involucrando la validez y confiabilidad. Esto pudiera servir para la toma de decisiones en relación al factor humano de las organizaciones. Cabe señalar, que la escala de clima organizacional puede servir como referente para la evaluación de diferentes problemáticas dentro de las instituciones orientadas al sector servicio, relacionando esta escala con cualquier otra variable que pudiera llegar a presentarse en la corporación, las cuales pudieran proveer de información para la toma de decisiones y salvaguardar los intereses de la organización y los trabajadores.

Una limitante para la elaboración del presente estudio fue el tamaño de la muestra, ya que debido a la dinámica de las instituciones, en una de ellas, no se tuvo la participación del total del personal. A su vez se propone probar el instrumento con una población que represente el sector servicio en la región y analizar la aplicación de las variables consideradas en este. De igual forma, se propone implementar medidas parecidas a otros sectores productivos con el fin de que en estos se cuente con instrumentos de evaluación confiables e instrumentos para la evaluación de sus condiciones. De la misma manera, es importante relacionar los indicadores de Clima Organizacional con el rendimiento laboral u algún otro indicador de producción del trabajador, con el fin de obtener validez predictiva.

El objetivo en las organizaciones de servicio es obtener un mejor desempeño de los trabajadores, pero si no se obtiene una medida confiable y válida que prediga el mejoramiento de esta práctica laboral, no se van a poder optimizar los recursos de la empresa y los servicios que se proporcionan a la comunidad. Esta herramienta nos permite elaborar un diagnóstico organizacional estableciendo, cuáles son las debilidades de la empresa para promover estrategias que permitan un mejor desempeño de la misma, que al final, repercute en la satisfacción de los usuarios del servicio, como se ha demostrado en otros estudios (Guerra et al., 2005; Peiró et al., 2002; Schmidt et al., 2001).

Se considera de suma importancia el ambiente laboral en el sector servicios ya que con esto los usuarios recibirían una mejor prestación. Un diagnóstico del clima organizacional permite introducir cambios planificados para poder intervenir en las áreas que existen problemas y promover un mejor ambiente laboral. 


\section{Referencias}

Anderson, D. Sweeny, D., y Williams, T. (2005). Estadística para Administración y Economía. Octava Edición, México D.F.: Math Learning

Bates, A.R. \& Khasawneh, S. (2007). Self efficacy and college students' perception and use of online learning systems. Computers in Human Behavior, 3(1), 175-191.

Cabrera, F. A. (2006). La Estadística, La ciencia de la Época. recuperado de http://www.monografias.com/trabajos40/laestadistica/la-estadistica.shtml el 09 de Marzo del 2007.

Chiavenato (2006) Gestión del Talento Humanos. Segunda Edición México: Mc. Graw Hill.

Chiavenato, A. (2002). Gestión del talento humano. Colombia.: Mc Graw Hill

Chiavenato, I. (2000). Administración de Recursos Humanos. Quinta Edición. Colombia.: Edit. Mc Graw Hill.

Cifre, E. (2008). Bienestar psicológico, características del trabajo y nuevas tecnologías: validación-ampliación del modelo vitamínico de Warr. Departamento de Psicología Evolutiva, Educativa, Social y Metodología. Recuperado de http:// biblioteca. universia.net/ficha.do?id=35130235, el marzo del 2007.

Corral, V., Frías, M. y González, D. (2001). Análisis Cuantitativo de Variables Latentes. Colección textos académicos, No. 13, Hermosillo, Sonora: Editorial UniSon.

Corral, V., Frías, M., y González, D. (2001). Análisis Cuantitativo de Variables Manifiestas. Colección textos académicos, No. 11, Hermosillo, Sonora: Editorial UniSon.

Del Castillo, T., Acosta, M. y Montes, A. (2008). Principales resultados del diagnóstico de clima laboral. Impacto en empresas seleccionadas de producción y servicios. Universidad de La Habana.: Master Business Administration, Recuperado de http://www.gestiopolis.com/organizaciontalento/estudio-de-clima-laboral.htm en Septiembre de 2008.

Farfan, C. y Cols. (2007). Evaluación y estudio del clima organizacional y la elaboración de un diagnostico correspondiente a recursos humanos. Secretaria de Agricultura, Ganadería, Desarrollo Rural, Pesca y Alimentación. PROYECTO INP-CS-AD/090/07, Recuperado de http://www.inp.sagarpa.gob.mx/Docs/Normateca/ EVALUACION\%20Y\%20ESTUDIO\%20DEL\%20CLIMA\%20 ORGANIZACIONAL\%20INP\%2017\%20DIC.pdf, en marzo del 2008.

Gan, F. (2005). La Comunicación en el Trabajo. Comunicación Organizacional. Recuperado de http://www.gestiopolis.com/ canales5/comerciohispano/63.htm el 12 de mayo del 2007

Gans, J. (1999). Limited information, the possibility of rational choice and the contingent valuation method. International Journal of Social Economics, 26, $402-414$

Guerra, J.M. Muñoz. H., Baron, M., Martinez, I., Munduate, L., (2006). Evaluación de factores de riesgo psicosocial: desarrollo de estrategias de detección y prevención del acoso laboral en las pymes. Sevilla: Consejería de Empleo de la Junta de Andalucía.
Guillen, C. y Guil, R. (2000). Psicología del Trabajo para relaciones laborales. Madrid: Edit. Mc Graw Hill.

Guizar, R. (2001). Desarrollo Organizacional, principios y aplicaciones. México. Edit. Mc Graw Hill.

Hudd, S.; Dumalao, J. y Erdman-Sager, D. (2000) Stress at college: effects of health habits, health status and self-esteem. College Student Journal, 34(2), 217-27.

Instituto Mexicano de Normalización y Certificación A.C. (2001). Sistemas de Gestión de la Calidad, Requisitos. Normas Mexicana ISO 9004:2000.

Maish, M. (2005). Estudios de Clima Organizacional. Recuperado de http://www.losrecursoshumanos.com/contenidos/290estudios-de-clima-organizacional.html, el 31 de mayo del 2008.

Maslach C. y Schaufeli, W. (1993). Historical and conceptual development of burnout. En: Schaufeli WB, Maslach C, Marek $\mathrm{T}$, (eds.). Professional burnout: Recent developments in theory and research. Londres: Taylor \& Francis, 1993: pp.1-16.

May, H.(2008).La Declaración sobre las Empresas Multinacionales. Trabajo, 62, Reduperado de http://www.ilo.org/Search3/ searchOnFast.do;jsesionid=0a038009cf3382303a85a194ea 884cddbabc5dea288.hkzFngTDp6WImQuUaNaLbhD3IN4Kxalah8S-xyIn3uKmAiN-AnwbQbxaNvzaAml-huKa30xgx95fj WTa3elpkzFngTDp6WImQuxah8LaN8Qc3yOb2b48OX3b4D tiOj1iyb8aMbynknvrkLOIQzNp65In0, en abril del 2008.

Meliá, N. (2008). ¿Cómo Evaluar los Riesgos Psicosociales en la Empresa? Metodologías, Oportunidades y Tendencias. Universidad de Valencia. Recuperado de www.uv.es/ seguridadlaboral, diciembre del 2008.

Melin, L. (1986). On the relationship of strategic change, leadership and organizational culture. Documento presentado a la Conferencia Internacional Strategic Management Society, Barcelona.

Montaño, J.L. (2003). ISO 9001 - 2000. Guía práctica de normas para implementarlas en la empresa. México: Edit. Trillas.

Moreno, I. (2001). Análisis del Factor Estrés en el Desarrollo Organizacional. Trabajo Escrito para obtener el título de Ingeniero Industrial y de Sistemas por la Universidad de Sonora.

Myers, D. (2000). Psicología Social, sexta edición. México.: Mc Graw Hill.

Peiro, J. M. (1999). Desencadenantes del estrés laboral. Valencia España: Univesitat du Valencia.

Pinazo, D.y Carrero, V. (2001). Lay Beliefs About Developing Countries in Relation to Helping Behaviors. Journal of Social Psychology, 150(4), 393-415.

Robbins, S. (2004). Comportamiento Organizacional. Decima edición. México: Pearson, Prentice Hill.

Rodriguez, D. (1999). Diagnóstico Organizacional. Tercera edición. Chile: Ediciones Universidad Católica de Chile.

Schmidt, Shulla, Schmitt, (2001) Psicología Industrial. México: Editorial Trillas.

Valenzuela, P.; Montenegro, V., Valenzuela, L.;\&Alvarado, S.(2007). Tipos de Investigaciones en las Ciencias Sociales., Centro de Investigación e Intervención Sistemática. Recuperado de http://www.psykhe.org/logospsykhe/2007/03012007c.htm. 